\title{
Abnormal Balloon Expulsion Test in Patients with Fecal Incontinence
}

\author{
Leila Neshatian' ${ }^{1}$, Chimi L. Fosso ${ }^{2}$, Eamonn M. Quigley ${ }^{1}$
}

1) Department of

Gastroenterology, Houston

Methodist, Weill Cornell

Medical College, Houston;

2) Internal Medicine

Department, Houston

Methodist, Weill Cornell

Medical College, Houston,

USA

Address for correspondence:

Leila Neshatian

Houston Methodist Hospital

6550 Fannin St Suite 1201,

Houston, TX 77030, USA

lneshati@stanford.edu
Received: 09.04.2020

Accepted: 20.05.2020

\begin{abstract}
Background \& Aims: Functional defecatory dysfunction is attributed to the pathophysiology of fecal incontinence (FI) in some patients. We hypothesized that patients with FI and abnormal balloon expulsion test (BET) have distinct manometric characteristics as compared to the patients with FI and normal BET. We aimed to compare the anorectal pressure profile in patients with FI, with or without abnormal BET and to identify risk factors associated with abnormal BET in FI.

Methods: We performed a retrospective review of 77 consecutive patients with ROME IV FI. Wilcoxon rank sum test, t-test, and Fisher exact tests were performed for comparison. Multivariable logistic regression was performed to identify factors associated with abnormal BET.

Results: Thirty-two percent of patients had abnormal BET. Demographics and surgical history and clinical symptoms, except for sensation of incomplete evacuation $(\mathrm{p}=0.02)$ and abdominal pain $(\mathrm{p}=0.03)$, were comparable in both groups. Anorectal pressure profile except for the median rectal propulsive pressures were similar between groups. Rectal propulsive pressures at simulated defecation were significantly lower in patients with abnormal BET $(\mathrm{p}=0.02)$. Mean sensory threshold for first sensation was also significantly higher in patients who had abnormal BET ( $\mathrm{p}=0.03$ ). Rectal propulsive pressures (OR: 1.03, 95\% CI: 1.00-1.06, $\mathrm{p}=0.032$ ) and rectal sensory threshold for first sensation (OR:0.94, 95\% CI: 0.90-0.99, p=0.02) were able to predict abnormal BET independently.

Conclusions: In patients with FI and similar clinical and anal pressure profile, rectal sensory threshold and rectal propulsive pressures at simulated defecation can determine normal BET.
\end{abstract}

Key words: fecal incontinence - functional defecatory dysfunction- balloon expulsion test - anorectal manometry.

Abbreviations: BET: balloon expulsion test; BMI: body mass index; FI: fecal incontinence; HR-ARM: highresolution anorectal manometry.

\section{INTRODUCTION}

Fecal incontinence (FI) is a common yet under-recognized problem that affects nearly $15 \%$ of community dwelling men and women [1]. It has a devastating impact on the affected individual's quality of life [2]. ROME IV, the diagnostic classification for functional gastrointestinal disorders, defines FI as recurrent, uncontrolled passage of fecal material for at least 3 months [3]. Despite the delineation of a number of risk factors for FI [advanced age, presence of diarrhea and urgency to defecate, high body mass index (BMI), cigarette smoking and presence of comorbid medical conditions] [4-6], the pathophysiology of FI remains poorly understood. Although a history of anorectal surgery, obstetric anal trauma sustained through the use of forceps or vacuum extraction, complicated episiotomy and presence of a rectocele have all been linked to the development of FI [4], anal sphincter dysfunction does not appear to be the principal pathology leading to loss of continence in many patients, and FI is considered to be a multifactorial disorder $[7,8]$.

Functional defecatory dysfunction with dyssynergic defecation has been described in some patients with fecal soiling or seepage. Indeed, Rao et al. [9] showed in a highly selective group of patients with fecal seepage, that more than $70 \%$ had a dyssynergic or obstructive pattern on anorectal 
manometry and that close to $30 \%$ had an abnormal balloon expulsion test (BET). While prevalence of FI seems to be similar between men and women [10], this form of FI has been reported more commonly among men [9]. Nonetheless, in studies of adult men with overt FI while only a minority had evidence of anal sphincter dysfunction, proctography revealed impaired rectal evacuation in $30 \%$ of these patients [11]. Those with evidence of rectal hyposensitivity were significantly more likely to have functional defecatory dysfunction [12]. Again, when compared to age-matched women with FI, functional defecatory dysfunction and rectal hyposensitivity were observed more frequently among males [11]. Overall, up to one-third of all patients with FI have no obvious sphincter pathology $[7,8]$. These patients with FI and normal anal sphincter function, seem to be younger, have more severe constipation and less severe FI. On manometry they are more likely to demonstrate a dyssynergic pattern of defecation and higher rectal defecation pressures $[8,13]$. Incomplete rectal evacuation has been associated with FI, not only in patients with normal anal sphincter function, but also in patients with a weak anal sphincter [7]. Overall, it appears that retained stools in the rectum increases the chance of leakage and, therefore, needs to be identified and corrected in patients with FI. Diagnosis of functional defecatory dysfunction in patients with FI is particularly important with regards to clinical management as these patients could be managed very effectively by biofeedback therapy $[14,15]$.

While functional defecatory dysfunction has been described among some patients with FI, the role of BET in patients with FI remains unclear. We hypothesized that patients with FI who have evidence of abnormal BET, have a distinctive anorectal pressure profile as compared to the ones with normal BET. Therefore, we sought to study differences in clinical and anorectal manometry parameters between patients with FI with regards to BET. Our study provides unique and much needed insight into the pathophysiology of FI which can lead to optimal clinical management of these patients.

\section{METHODS}

We reviewed records of 77 consecutive patients who had undergone high-resolution anorectal manometry (HRARM) at our motility laboratory for the diagnosis of FI based on ROME IV criteria, from November 2015 to July 2017. The Institutional Review Board approved the retrospective protocol. Patients younger than 18 years were excluded. Relevant data were collected on each patient, including age, gender, BMI as well as clinical information.

Anorectal pressures were assessed using a high-resolution catheter (Medtronic, Minneapolis, MN, USA) in the left lateral position. All patients were instructed to use an enema 1 hour prior to the anorectal manometry procedure and digital rectal exam was performed prior to the test to ensure an empty rectum. A high-resolution catheter comprises 10 circumferential sensors with 8 of them arrayed at $6 \mathrm{~mm}$ intervals along the anal canal and a further 2 sensors in the rectal balloon. The average pressure that was obtained from the sensors at each section resulted in the mean value of the anal and rectal pressures. Rectal propulsive pressures were resulted from the proximal sensor located in the rectal balloon, and the average of pressures from anal sensors through the e-sleeve provided the anal pressures.

Protocol comprised of the assessment of anorectal pressures at rest, during squeeze, and simulated defecation with an empty rectal balloon. Resting pressures were recorded after 3 to 5 minutes of acclimation to the catheter. Squeeze pressures were obtained twice, 20 seconds of holding the squeeze and 1 minute of rest between the 2 squeezes. Then, patients were instructed to push and bear down to simulate evacuation for 20 seconds, twice with 1-minute interval resting period.

Thereafter, rectoanal inhibitory reflex and rectal sensory function were simultaneously evaluated by progressively distending a rectal balloon in $10 \mathrm{ml}$ increments to reach the patient's first sensory threshold and thereafter in $30 \mathrm{ml}$ increments until a maximum volume of $350 \mathrm{ml}$ or until patients reported severe urgency or discomfort. Manoview, AR v3.0 was used to analyze the anorectal pressures. Rectal sensitivity was determined based on normal values previously obtained from healthy volunteers [16]. Patients were characterized as having abnormal sensory patterns if they had 2 consecutive abnormal sensory thresholds.

\section{Balloon expulsion testing}

Balloon expulsion was performed using a non-latex rectal balloon (Mui Scientific, Mississauga, Canada) filled with 50 $\mathrm{mL}$ of water. Patients were asked to expel the balloon while sitting on a commode in privacy. If subjects could not expel the balloon in 2 minutes, it was deflated and removed manually.

\section{Statistical Analysis}

Demographics, past surgical history and risk factors, coexisting symptoms, and anorectal physiology results were extracted for all subjects. Baseline characteristics and ARM parameters (shown as median and interquartile ranges) of patients with or without a normal BET were compared, using the Wilcoxon rank-sum test. Rectal sensory thresholds are shown as mean and SEM and compared using the Student t-test. Categorical statistical analysis was performed by a Fisher exact probability test. We used multivariate logistic regression to evaluate the association between ARM parameters and odds of abnormal BET (Stata 14, College Station, TX, USA). Statistical significance was defined by a p value of less than 0.05 .

\section{RESULTS}

\section{Demographic}

We reviewed clinical data from a total of 77 consecutive patients (7 men), with ROME IV FI, who had undergone HR-ARM. Thirty two percent (25/77) of patients with FI had an abnormal BET. Age, BMI, history of previous anorectal and obstetrical surgeries of patients who passed BET were comparable to patients who had abnormal BET. Demographic features are presented in Table I. Patients with abnormal BET were more likely to report a sensation of incomplete evacuation $(60 \%$ vs $33 \%, \mathrm{p}=0.02)$ and abdominal pain $(64 \%$ vs $38 \%, \mathrm{p}=0.03)$. 
Table I. Demographics based on the Results of Rectal Balloon Expulsion Test

\begin{tabular}{lccc}
\hline Demographic Variables & Normal BET & Abnormal BET & $\mathrm{p}$ \\
\hline Participants, $\mathrm{n}$ & $52(4$ male $)$ & $25(3 \mathrm{male})$ & \\
Age, years, median (IQR) & $62.50(54.00-72.50)$ & $57.00(52.00-67.00)$ & 0.12 \\
BMI, kg/m2, median (IQR) & $27.80(22.93-30.70)$ & $28.80(25.30-33.78)$ & 0.33 \\
No of vaginal deliveries, median (IQR) & $2(0-3)$ & $2(0-3)$ & 0.51 \\
Episiotomy, n (\%) & $19(36)$ & $8(32)$ & 0.42 \\
Anorectal surgeries, $\mathrm{n}(\%)$ & $7(13)$ & $6(24)$ & 0.36 \\
No of BM/day, median (IQR) & $1.50(0.71-3.00)$ & $1.00(2.00-4.00)$ & 0.20 \\
Passive incontinence, $\mathrm{n}(\%)$ & $34(65)$ & $16(64)$ & 0.55 \\
Gas incontinence, $\mathrm{n}(\%)$ & $31(60)$ & $9(36)$ & 0.05 \\
Liquid incontinence, $\mathrm{n}(\%)$ & $33(63)$ & $19(76)$ & 0.31 \\
Solid stool incontinence, $\mathrm{n}(\%)$ & $19(36)$ & $4(16)$ & 0.06 \\
Abdominal bloating, $\mathrm{n}(\%)$ & $32(61)$ & $12(48)$ & 0.32 \\
Abdominal pain, $\mathrm{n}(\%)$ & $20(38)$ & $16(64)$ & 0.03 \\
Concurrent constipation, $\mathrm{n}(\%)$ & $13(25)$ & $9(36)$ & 0.23 \\
Use of laxatives, $\mathrm{n}(\%)$ & $15(28)$ & $6(24)$ & 0.43 \\
Incomplete evacuation, $\mathrm{n}(\%)$ & $17(33)$ & $15(60)$ & 0.02 \\
\hline
\end{tabular}

BET: balloon expulsion test; BM: bowel movements; BMI: body mass index.

\section{Anorectal pressure profile according to the balloon expulsion testing \\ The prevalence of a dyssynergic pattern on HR-ARM, based on paradoxical anal contraction or incomplete relaxation, during simulated defecation, was $81 \%(63 / 77)$ with 9 patients fulfilling the ROME IV criteria for functional defecatory dysfunction. Anorectal pressure parameters and rectal sensory thresholds for those with or without a normal BET are compared in Table II. During simulated defecation, patients with an abnormal BET had significantly lower rectal propulsive pressures (Fig. 1).}

\section{Rectal Sensation in Patients with Abnormal Balloon} Expulsion Test

There was a significant difference in the mean sensory threshold for first sensation in patients with abnormal BET as

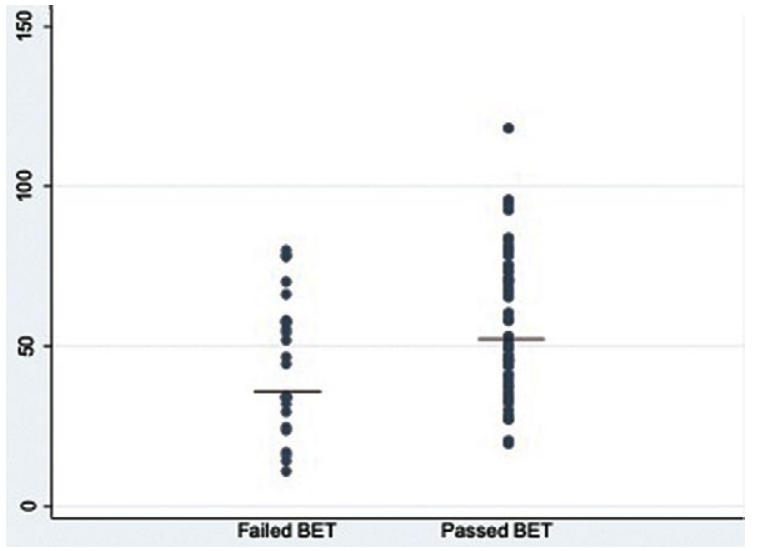

Fig. 1. Rectal propulsive pressures and balloon expulsion test (line shows the median).

Table II. Anorectal parameters according to the balloon expulsion test (Median and interquartile range)

\begin{tabular}{lccc}
\hline Anorectal profile & $\begin{array}{c}\text { Normal BET (n:52) } \\
\text { Median (IQR) }\end{array}$ & $\begin{array}{c}\text { Abnormal BET (n:25) } \\
\text { Median (IQR) }\end{array}$ & $\mathrm{p}$ \\
\hline Rest & & & \\
\hline Mean resting anal pressure (mmHg) & $52.15(41.65-76.45)$ & $68.30(51.60-78.20)$ & 0.13 \\
Mean resting rectal pressure (mmHg) & $49.30(34.40-69.90)$ & $65.60(45.60-77.60)$ & 0.15 \\
\hline Squeeze & & & \\
\hline Squeeze anal pressure (mmHg) & $109.45(75.70-199.25)$ & $146.80(117.40-169.10)$ & 0.08 \\
Length of anal HPZ (cm) & $3.00(2.20-3.75)$ & $2.80(2.10-3.20)$ & 0.35 \\
Duration of sustained squeeze (sec) & $19.75(7.95-20.05)$ & $16.10(6.30-20.00)$ & 0.52 \\
\hline Simulated defecation & & & 0.69 \\
\hline Anal pressure (mmHg) & $76.45(51.90-94.40)$ & $78.30(61.20-96.30)$ & 0.41 \\
Anal relaxation (\%) & $-21.91(-82.33-10.19)$ & $-13.24(-36.38-25.07)$ & 0.02 \\
Rectal propulsive pressure (mmHg) & $52.15(39.70-73.25)$ & $44.30(29.20-57.80)$ & 0.06 \\
Rectoanal gradient (mmHg) & $-16.90(-37.90-2.10)$ & $-34.90(-42.50--11.20)$ & $<0.001$ \\
Functional defecation dysfunction, $\mathrm{n}$ & 0 & 9 &
\end{tabular}

BET: balloon expulsion test; HPZ: high pressure zone. 
compared to the others $[19( \pm 2)$ vs. $29( \pm 5), p=0.03]$. Despite variances in volumes required to trigger urge sensation and maximum tolerable volumes in patients with abnormal BET, differences were not statistically significant (Table III). Twenty five patients with normal BET and 8 patients with abnormal BET had impaired rectal sensation ( $\geq 2$ sensory thresholds above normal published values [16]). All patients had an intact rectoanal inhibitory reflex.

Table III. Sensory thresholds parameters according to the balloon expulsion test (mean \pm SEM)

\begin{tabular}{lccc}
\hline $\begin{array}{l}\text { Rectal sensory } \\
\text { thresholds }\end{array}$ & $\begin{array}{c}\text { Normal BET } \\
(\mathrm{n}: 52) \text { mean } \\
(\text { SEM })\end{array}$ & $\begin{array}{c}\text { Abnormal BET } \\
(\mathrm{n}: 25) \text { mean } \\
(\text { SEM })\end{array}$ & $\mathrm{p}$ \\
\hline First sensation $(\mathrm{ml})$ & $19( \pm 2)$ & $29( \pm 5)$ & 0.03 \\
Urge sensation $(\mathrm{ml})$ & $92( \pm 6)$ & $97( \pm 14)$ & 0.75 \\
$\begin{array}{l}\text { Maximal tolerable } \\
\text { volume }(\mathrm{ml})\end{array}$ & $142( \pm 8)$ & $136( \pm 13)$ & 0.69 \\
\hline
\end{tabular}

Predictors of balloon expulsion test in patients with fecal incontinent

In multiple logistic regression analysis (Table IV), the presence of lower rectal propulsive pressures during simulated defecation was an independent risk factor for an abnormal BET while a higher anal sensory threshold for first sensation predicted successful balloon expulsion. In contrast, neither rectal or anal resting nor squeeze pressures, lower residual anal pressures and greater anal relaxation at simulated defecation predicted normal BET. Rectal sensory thresholds for urge to defecate or maximum tolerated volumes were also not predictive of normal BET.

Table IV. Multiple variable logistic regression models for the rectal balloon expulsion test.

\begin{tabular}{lccc}
\hline & $\begin{array}{c}\text { Odds } \\
\text { Ratio }\end{array}$ & $\mathrm{p}$ & [95\% Conf. Interval] \\
\hline BMI & 0.96 & 0.40 & $0.87-1.05$ \\
Sphincter Length & 1.57 & 0.16 & $82-3.01$ \\
Duration of squeeze & 1.03 & 0.42 & $0.95-1.12$ \\
Residual anal pressure & 0.99 & 0.32 & $0.97-1.0$ \\
Percent anal relaxation & 1.00 & 0.60 & $0.99-1.00$ \\
Rectal propulsive & 1.03 & $\mathbf{0 . 0 3}$ & $1.00-1.06$ \\
pressure & & & $0.90-0.99$ \\
First sensation & 0.94 & $\mathbf{0 . 0 2}$ & $0.97-1.01$ \\
Urge to defecate & 0.99 & 0.82 & $0.99-1.02$ \\
Max tolerable volume & 1.01 & 0.12 & \\
\hline
\end{tabular}

\section{DISCUSSION}

Functional defecatory dysfunction is a well described abnormality in patients with FI [6]. Identifying clinical and anorectal characteristics that are strongly associated with functional defecatory dysfunction in FI is important as they could determine the management strategy $[2,14,15]$. Our study demonstrated, first, that up to $32 \%$ of patients with diagnosis of FI, based on ROME IV criteria, had abnormal BET. Second, despite comparable clinical profile and anal pressure profile, distinct differences exist in rectal propulsive forces and sensory profiles among FI patients with or without an abnormal BET. And third, that rectal propulsive pressures at simulated defecation and rectal sensory threshold for first sensation can independently predict BET results in patients with FI.

Anorectal manometry is recommended as the initial test, preceding anal imaging, for the evaluation of patients with FI who have failed conservative management [15]. Balloon expulsion test, routinely done in conjunction with anorectal manometry, has a high positive predictive value for a diagnosis of functional defecatory dysfunction [17]. Even though BET is a valuable test for the evaluation of functional defecatory dysfunction, its utility in patients with FI is not clear. This, at least in part, stems from the notion that FI is generally presumed to be a low anal sphincter pressure state, in which balloon expulsion should not pose any challenge. To our surprise, $32 \%$ of our patients who had undergone anorectal manometry and BET for a ROME IV diagnosis of FI proved to have abnormal BET. Abnormal BET was noted despite comparable symptoms and baseline anal sphincter pressures at rest and squeeze, as well as simulated defecation.

Bowel disturbances are described as one of the strongest independent risk factors for FI, in the community $[5,18]$. While loose stool or diarrhea will be inclined to leak easily, other bowel disturbances, particularly constipation, seem to be rather common [2], and reported in up to $60 \%$ of patients with FI [8]. Not surprisingly, FI patients with normal anal function have more severe constipation related to functional defecatory dysfunction [8]. In our study, while the average number of bowel movements was comparable between groups, $25 \%$ of patients with normal BET and $36 \%$ of patients with abnormal BET had reported the presence of concurrent constipation, with a comparable need for laxative use. Consistent with previous studies, the liquid stool was the most common form of incontinent stool in both groups.

The presence of a sensation of incomplete evacuation has been reported previously in patients with FI. Indeed, a sense of incomplete evacuation is an established risk factor for FI [18]. In a cross-sectional population-based survey, self-reported sensation of incomplete evacuation robustly predicted the risk of developing FI (OR:3.4) [19]. Yet, the relationship between the sensation of incomplete evacuation and abnormal BET, in FI, has not been previously studied. We found that a sensation of incomplete evacuation was reported by $60 \%(15 / 25)$ of patients with FI who had an abnormal BET as compared to only $33 \%(17 / 52)$ of patients with normal BET $(p=0.02)$. Also, we found that patients with an abnormal BET were more likely to complain of abdominal pain. This is likely to be explained by the presence of other concurrent functional pathologies such as irritable bowel syndrome and colonic retention of the stool, colonic distention and therefore abdominal distension and pain as described with other delayed colonic motility pathologies [20].

Passive incontinence or passage of stool without awareness is typically associated with an incompetent anal sphincter [21]. Alterations of rectal compliance, capacity, and rectoanal sensation have been documented as the culprit of this phenomenon in some patients [22]. On the other hand, in patients with severe constipation, overflow incontinence will 
be associated with the symptoms of passive incontinence. We found that around $65 \%$ of our patients, regardless of BET results, had reported the presence of passive incontinence. Given the fact that anal pressures at rest, which mainly reflects internal anal sphincter, were overall preserved in our patients with abnormal BET. Incomplete rectal evacuation is presumed to cause passive leakage of stool in this group, whereas in patients with normal BET, resting anal sphincter pressures were slightly but not significantly lower. Whether sphincter impairment along with other rectal sensory dysfunction could be attributed to passive incontinence in patients with normal BET will require further investigation that was not in the scope of our study.

Rao et al. [9], have suggested that dyssynergic defecation is an important factor in the pathophysiology of fecal seepage. They showed that in comparison to those with overt incontinence, patients with fecal seepage had significantly higher residual anal pressures, but similar rectal propulsive pressures, during simulated defecation. Nine patients in this cohort had symptoms of constipation with anorectal manometry and BET confirming the ROME IV diagnosis of functional defecatory dysfunction. Functional defecation dysfunction is a well-recognized etiology of FI in some patients. Patients with functional defecation dysfunction and FI may have an impaired rectal sensation despite normal pudendal nerve function and sphincter function [9]. We also noted that patients with FI and functional defecatory dysfunction had significantly higher resting anorectal pressures as well as anal squeeze pressures as compared to patients with FI alone. The rectal sensory levels were comparable in this cohort between the groups (data not shown).

In contrast, in our study, rectal propulsive pressures during simulated defecation were significantly lower in patients with an abnormal BET as compared to normal BET, regardless of functional defecatory dysfunction. This was despite comparable residual anal pressures and rate of anal relaxation between the two groups. These findings underscore the point that impaired rectal pressures might be the underlying pathology in patients with FI and abnormal BET. Therefore, the mechanism of abnormal BET appears to be quite different in patients with FI as compared to those with FI and concurrent functional defecatory dysfunction.

In a recent study, abnormal BET (> 60 seconds) was reported in $15 \%$ of asymptomatic healthy females and $6 \%$ of asymptomatic healthy males [23]. Healthy women with an abnormal BET were found to also have a lower rectoanal gradient during evacuation. Future studies should further evaluate whether the abnormal BET in healthy controls and FI patients is related to abnormal structural pathologies such as rectocele.

Abnormal rectal evacuation is a known etiology of FI, as the retained stool in the rectum is prone to induce leakage. Functional defecatory dysfunction has been also described as an important pathophysiological mechanism in those patients with FI who have normal anal sphincter function $[8,11,13]$. This category has been described in up to $36 \%$ of men [11] and 13-15\% of women [13] with FI. In our study, median anal resting tone and anal squeeze pressures were lower than normal overall. However, in some patients, anal sphincter function was preserved as evidenced by normal anal pressures at rest and on squeeze. Among multiple anorectal parameters studied, only rectal propulsive pressures during simulated defecation were significantly lower in patients with abnormal BET. Nevertheless, in previous observations of patients with FI despite normal anal sphincter function, rectal propulsive pressures were significantly elevated at simulated defecation, indicating straining in these patients. Therefore, the primary mechanism of functional defecatory disorder in patients with normal sphincter function is quite distinct from that of our patients, where lower propulsive forces were the cause of impaired rectal evacuation. This highlights the fact that normal rectal evacuation requires coordinated anorectal function including both adequate rectal propulsive pressures and proper anal relaxation. Indeed, the only risk factor associated with abnormal BET, based on logistic regression, was a lower level of rectal pressure on simulated defecation analysis (Table IV). It is intuitive that in patients with comparable anal sphincter function the BET would rely mainly on rectal/intraabdominal pressures. And our study demonstrated that lower rectal propulsive pressures at simulated defecation was an independent risk factor for an abnormal BET and functional defecatory dysfunction in patients with FI.

Prevalence of dyssynergia on anorectal recordings was $81 \%$, in our study, with type I dyssynergia being the most common subtype in this cohort; no differences in relative prevalence of the various subtypes of dyssynergia were seen between those with or without normal BET. In other studies, patients with seepage were found to have a higher rate of dyssynergia (72\%) as compared to patients with overt FI (30\%) [9]. Dyssynergia was also reported in $71 \%$ of women with normal sphincter function FI [13]. Indeed, dyssynergia with preserved rectal propulsive pressures (type I and IV dyssynergia), was identified as an important risk factor for FI in women with normal anal sphincter function [13]. This was despite comparable rates of abnormal BET between those with normal and impaired anal sphincter pressures. It must be conceded, nevertheless, that clinical utility of HR-ARM in diagnosis of dyssynergia has been questioned, as "dyssynergia", as defined by manometry, seems to be a common finding among healthy individuals [24]. In one study, more than $90 \%$ of healthy controls showed evidence of dyssynergia on anorectal manometric recordings. Indeed, only Types III and IV proved to be discriminatory between those with normal and abnormal balloon expulsion times [25]. Therefore, relevance of findings of isolated dyssynergia to FI deserves further assessment.

Previous studies have shown that rectal sensation seems to be important to achieve effective rectal evacuation $[6,11]$. Sensory retraining impacts the outcome of biofeedback in patients with FI [26]. It has been shown that FI patients with enhanced rectal sensory thresholds are less likely to respond to biofeedback therapy as compared to patients with normal rectal sensation [2]. A comparable number of our patients in each group had rectal hyposensitivity at $\geq 2$ sensory thresholds above normal values and therefore, enhanced rectal sensory threshold for first sensation could indeed determine abnormal BET in patients with FI.

Limitations to our study include a relatively small sample size, its retrospective nature, and the lack of utilizing a validated 
FI severity scale as well as anorectal imaging. Future prospective studies with validated questionnaires and employing dynamic imaging of anorectum and pelvic floor will help to further reveal the pathophysiology of FI.

\section{CONCLUSIONS}

We showed that one third of patients with Rome IV diagnosis of FI have abnormal BET, despite the comparable clinical and anal pressure profile. We found differences in rectal properties of patients with FI who had abnormal BET. Indeed, rectal first sensory threshold and rectal propulsive pressures are the independent risk factors for abnormal BET in patients with FI.

Our findings provide important novel data on the pathophysiology of FI. This work is the first to provide evidence for the rectal propulsive forces as the mechanism of abnormal BET in patients with FI. Future prospective studies to replicate these findings on larger patient cohorts will be required.

Conflicts of interest: None to declare.

Authors' contribution: L.N. designed the study, analyzed the data and drafted the manuscript. C.L.F. contributed to the data collection. E.M.Q. contributed to the conception and interpretation of data. E.M.Q. and C.L.F. critically revised the manuscript. All authors approved the final version of the article

Acknowledgment: This work was supported in part by a gift from the Hughes Sterling Foundation.

\section{REFERENCES}

1. Menees SB, Almario CV, Spiegel BMR, Chey WD. Prevalence of and Factors Associated With Fecal Incontinence: Results From a PopulationBased Survey. Gastroenterology 2018;154:1672-1681.e3. doi:10.1053/j. gastro.2018.01.062

2. Bedard K, Heymen S, Palsson OS, Bharucha AE, Whitehead WE. Relationship between symptoms and quality of life in fecal incontinence. Neurogastroenterol Motil 2018;30:10.1111/nmo.13241. doi:10.1111/ nmo.13241

3. Rao SS, Bharucha AE, Chiarioni G, et al. Functional Anorectal Disorders. Gastroenterology 2016 Mar 25. doi:10.1053/j. gastro.2016.02.009

4. Bharucha AE, Fletcher JG, Melton LJ 3rd, Zinsmeister AR. Obstetric trauma, pelvic floor injury and fecal incontinence: a population-based case-control study. Am J Gastroenterol 2012;107:902-911. doi:10.1038/ ajg. 2012.45

5. Bharucha AE, Zinsmeister AR, Schleck CD, Melton LJ 3rd. Bowel disturbances are the most important risk factors for late onset fecal incontinence: a population-based case-control study in women. Gastroenterology 2010;139:1559-1566. doi:10.1053/j. gastro.2010.07.056

6. Bharucha AE, Dunivan G, Goode PS, et al. Epidemiology, pathophysiology, and classification of fecal incontinence: state of the science summary for the National Institute of Diabetes and Digestive and Kidney Diseases (NIDDK) workshop. Am J Gastroenterol 2015;110:127-136. doi:10.1038/ajg.2014.396
7. Bharucha AE, Fletcher JG, Harper CM, et al. Relationship between symptoms and disordered continence mechanisms in women with idiopathic faecal incontinence. Gut 2005;54:546-555. doi:10.1136/ gut.2004.047696

8. Brochard C, Bouguen G, Bodere A, et al. Prospective cohort study of phenotypic variation based on an anal sphincter function in adults with fecal incontinence. Neurogastroenterol Motil 2016;28:1554-160. doi:10.1111/nmo.12855

9. Rao SS, Ozturk R, Stessman M. Investigation of the pathophysiology of fecal seepage. Am J Gastroenterol 2004;99:2204-2209.

10. Whitehead WE, Borrud L, Goode PS, et al. Fecal incontinence in US adults: epidemiology and risk factors. Gastroenterology 2009;137:512517.e2. doi:10.1053/j.gastro.2009.04.054

11. Townsend DC, Carrington EV, Grossi U, et al. Pathophysiology of fecal incontinence differs between men and women: a case-matched study in 200 patients. Neurogastroenterol Motil 2016;28:1580-1588. doi:10.1111/nmo.12858

12. Burgell RE, Bhan C, Lunniss PJ, Scott SM. Fecal incontinence in men: coexistent constipation and impact of rectal hyposensitivity. Dis Colon Rectum 2012;55:18-25. doi:10.1097/DCR.0b013e318237f37d

13. James-Stevenson T, Xu H, Heit M, Shin A. Age and Dyssynergia Subtypes Associated With Normal Sphincter Pressures in Women With Fecal Incontinence. Female Pelvic Med Reconstr Surg 2018;24:247-251. doi:10.1097/SPV.0000000000000415

14. Rao SSC, Benninga MA, Bharucha AE, Chiarioni G, Di Lorenzo C, Whitehead WE. ANMS-ESNM position paper and consensus guidelines on biofeedback therapy for anorectal disorders. Neurogastroenterol Motil 2015;27:594-609. doi:10.1111/nmo.12520

15. Whitehead WE, Rao SS, Lowry A, et al. Treatment of fecal incontinence state of the science summary for the National Institute of Diabetes and Digestive and Kidney Diseases workshop. Am J Gastroenterol 2015;110:138-146. doi:10.1038/ajg.2014.303

16. Noelting J, Ratuapli SK, Bharucha AE, Harvey DM, Ravi K, Zinsmeister AR. Normal values for high-resolution anorectal manometry in healthy women: effects of age and significance of rectoanal gradient. Am J Gastroenterol 2012;107:1530-1536. doi:10.1038/ajg.2012.221

17. Chiarioni G, Kim SM, Vantini I, Whitehead WE. Validation of the balloon evacuation test: reproducibility and agreement with findings from anorectal manometry and electromyography. Clin Gastroenterol Hepatol 2014;12:2049-2054. doi:10.1016/j.cgh.2014.03.013

18. Bharucha AE, Seide BM, Zinsmeister AR, Melton LJ 3rd. Relation of bowel habits to fecal incontinence in women. Am J Gastroenterol 2008;103:1470-1475.

19. Rey E, Choung RS, Schleck CD, Zinsmeister AR, Locke GR 3rd, Talley NJ. Onset and risk factors for fecal incontinence in a US community. Am J Gastroenterol 2010;105:412-419. doi:10.1038/ajg.2009.594

20. Bharucha AE, Chakraborty S, Sletten CD. Common Functional Gastroenterological Disorders Associated With Abdominal Pain. Mayo Clin Proc 2016;91:1118-1132. doi:10.1016/j.mayocp.2016.06.003

21. Bharucha AE, Zinsmeister AR, Locke GR, et al. Risk factors for fecal incontinence: a population-based study in women. Am J Gastroenterol 2006;101:1305-1312.

22. Salvioli B, Bharucha AE, Rath-Harvey D, Pemberton JH, Phillips SF. Rectal compliance, capacity, and rectoanal sensation in fecal incontinence. Am J Gastroenterol 2001;96:2158-1268.

23. Oblizajek NR, Gandhi S, Sharma M, et al. Anorectal pressures measured with high-resolution manometry in healthy people-Normal values and asymptomatic pelvic floor dysfunction. Neurogastroenterol Motil 2019;31:e13597. doi:10.1111/nmo.13597 
24. Grossi U, Carrington EV, Bharucha AE, Horrocks EJ, Scott SM, Knowles CH. Diagnostic accuracy study of anorectal manometry for diagnosis of dyssynergic defecation. Gut 2016;65:447-455. doi:10.1136/ gutjnl-2014-308835

25. Ratuapli SK, Bharucha AE, Noelting J, Harvey DM, Zinsmeister AR. Phenotypic identification and classification of functional defecatory disorders using high-resolution anorectal manometry. Gastroenterology 2013;144:314-322.e2. doi:10.1053/j.gastro.2012.10.049

26. Chiarioni G, Bassotti G, Stanganini S, Vantini I, Whitehead WE. Sensory retraining is key to biofeedback therapy for formed stool fecal incontinence. Am J Gastroenterol 2002;97:109-117. 